\title{
Can Mobile Phones Improve Learning? Evidence from a Field Experiment in Niger
}

Jenny C. Aker, Christopher Ksoll and Travis J. Lybbert

\section{Web Appendix}

\section{Table A1: Attrition and Test Absenteeism}

\begin{tabular}{|c|c|c|c|}
\hline & $\mathbf{A B C}$ & Non-ABC & \\
\hline & $\begin{array}{l}\text { Mean } \\
\text { (s.d.) }\end{array}$ & $\begin{array}{l}\text { Mean } \\
\text { (s.d.) }\end{array}$ & $\begin{array}{l}\text { Difference } \\
\text { Coeff (s.e.) }\end{array}$ \\
\hline & $(1)$ & $(2)$ & (3) \\
\hline \multicolumn{4}{|l|}{ Panel A: Drop-Out } \\
\hline Pre-ABC Module & $\begin{array}{l}.042 \\
(.2)\end{array}$ & $\begin{array}{c}.035 \\
(.184)\end{array}$ & $\begin{array}{c}-.01 \\
(.015)\end{array}$ \\
\hline Post-ABC Module & $\begin{array}{l}.036 \\
(.186)\end{array}$ & $\begin{array}{c}.06 \\
(.238)\end{array}$ & $\begin{array}{l}-0.02 \\
(.02)\end{array}$ \\
\hline \multicolumn{4}{|l|}{ Panel B: June Test Rounds (Immediate) } \\
\hline Absenteeism (absent day of test $=1$ ) & $\begin{array}{l}0.20 \\
(.4)\end{array}$ & $\begin{array}{c}0.19 \\
(.394)\end{array}$ & $\begin{array}{l}0.000 \\
(.018)\end{array}$ \\
\hline Age of absentee & $\begin{array}{c}34.13 \\
(11.26)\end{array}$ & $\begin{array}{c}37.48 \\
(12.088)\end{array}$ & $\begin{array}{l}-1.32 \\
(0.92)\end{array}$ \\
\hline Gender of absentee $($ female $=1)$ & $\begin{array}{c}0.49 \\
(.5) \\
\end{array}$ & $\begin{array}{c}.356 \\
(.479) \\
\end{array}$ & $\begin{array}{c}0.14 * * * \\
(0.03)\end{array}$ \\
\hline \multicolumn{4}{|l|}{ Panel C: January Test Rounds (Persistent) } \\
\hline Absenteeism (absent day of test=1) & $\begin{array}{c}0.31 \\
(.463)\end{array}$ & $\begin{array}{c}0.30 \\
(.454)\end{array}$ & $\begin{array}{l}-0.012 \\
(.018)\end{array}$ \\
\hline Age of absentee & $\begin{array}{c}34.25 \\
(11.67)\end{array}$ & $\begin{array}{c}36.06 \\
(12.61)\end{array}$ & $\begin{array}{l}-0.94 \\
(0.94)\end{array}$ \\
\hline Gender of absentee $($ female $=1)$ & $\begin{array}{c}0.44 \\
(.496)\end{array}$ & $\begin{array}{l}.401 \\
(.49)\end{array}$ & $\begin{array}{c}0.03 \\
(0.02)\end{array}$ \\
\hline
\end{tabular}

Notes: Column 1 presents the mean for ABC villages, Column 2 presents the mean for non-ABC villages. Column 3 reports the coefficient from a regression of the dependent variable on an $\mathrm{ABC}$ indicator variable and sub-region fixed effects to account for randomization, and so does not exactly equal the difference between Columns 1 and 2 . HuberWhite standard errors clustered at the village level presented in parentheses. $* * *, * *, *$ denote statistical significance at the $1,5,10$ percent levels, respectively. 
Table A2: Comparison of Teacher Characteristics by Year

\begin{tabular}{|c|c|c|c|}
\hline & ABC & Non-ABC & \\
\hline & $\begin{array}{l}\text { Mean } \\
\text { (s.d.) }\end{array}$ & $\begin{array}{c}\text { Mean } \\
\text { (s.d.) }\end{array}$ & $\begin{array}{l}\text { Difference } \\
\text { Coeff (s.e.) }\end{array}$ \\
\hline & $(1)$ & (2) & (3) \\
\hline Panel A: Teacher-Level Characteris & & & \\
\hline Education (number of years) & 8.86 & 8.25 & 0.61 \\
\hline & $(1.315)$ & $(2.286)$ & $(0.39)$ \\
\hline Age & 32.25 & 33.07 & -0.82 \\
\hline & $(6.65)$ & $(9.626)$ & $(1.82)$ \\
\hline Gender $($ Female $=1)$ & .345 & .254 & 0.09 \\
\hline & $(.479)$ & $(.439)$ & $(0.09)$ \\
\hline Local $($ Teacher from village $=1)$ & .667 & .763 & -0.10 \\
\hline & $(.475)$ & $(.429)$ & $(0.11)$ \\
\hline Number of observations & 60 & 59 & 119 \\
\hline Panel B: Teacher-Level Characteris & & & \\
\hline Education (number of years) & 8.431 & 8.362 & -0.07 \\
\hline & $(1.957)$ & $(1.972)$ & $(0.26)$ \\
\hline Age & 32.94 & 33.048 & -0.11 \\
\hline & $(8.697)$ & $(8.929)$ & $(1.32)$ \\
\hline Gender $($ Female $=1)$ & .379 & .352 & 0.03 \\
\hline & $(.487)$ & $(.48)$ & $(0.05)$ \\
\hline Local $($ Teacher from village $=1)$ & .69 & .755 & -0.07 \\
\hline & $(.465)$ & $(.432)$ & $(0.07)$ \\
\hline Number of observations & 116 & 110 & 226 \\
\hline
\end{tabular}

Notes: Column 1 presents the mean for $\mathrm{ABC}$ villages, Column 2 presents the mean for non- $\mathrm{ABC}$ villages. Column 3 reports the coefficient from a regression of the variable on an indicator variable for $\mathrm{ABC}$, but does not include sub-region fixed effects to account for randomization due to a limited number of observations. Huber-White standard errors clustered at the village level presented in parentheses. $*^{* *}, * *, *$ denote statistical significance at the $1,5,10$ percent levels, respectively. 
Table A3: Impact of the ABC Program on Test Scores: Alternative Specifications

Panel A: Writing Z-Scores Simple Difference

Value Added

Alternative Normalization

(1)

(2)

(3)

(4)

(5)

(6)

$\mathrm{ABC}$

$\begin{array}{ll}0.149 * & 0.132 * \\ (0.079) & (0.073)\end{array}$

$0.157 *$

$0.142 *$

$-0.020$

$-0.156$

Baseline Test Z-score

(0.080)

(0.074)

$(0.094)$

(0.162)

ABC*Post

$(0.018)$

$0.087 * * *$

$0.812 * * *$

2009 Cohort

(0.018)

(0.238)

$0.819^{*}$

$0.860^{*}$

$(0.439)$

(0.435)

$-0.058$

0.000

$-0.073$

$-0.013$

$0.812 * * *$

$0.947 * * *$

Female

$-0.649 * * *$

(0.077)

(0.085)

(0.078)

(0.238)

(0.214)

Female

$$
\text { (0.044) }
$$

(0.045)

$-0.638 * * *$

$-0.644 * * *$

$-1.998 * * *$

$-1.989 * * *$

Age

$-0.015^{* * *}$

$-0.016 * * *$

(0.043)

(0.043)

(0.142)

(0.141)

(0.002)

(0.002)

$-0.015^{* * *}$

$-0.016^{* * *}$

$-0.053 * * *$

$-0.051 * * *$

Sub-region fixed effects

No

(0.002)

(0.002)

(0.005)

(0.005)

Number of observations

7,148

Yes

No

Yes

6,912

6,912

No

Yes

$\mathrm{R}^{2}$

0.123

7,148

0.133

0.182

12,823

12,823

0.174

0.416

0.436

\section{Panel B: Math Z-Scores}

ABC

$0.172 * *$

$0.129 *$

$0.185 * *$

$0.144 * *$

$-0.090$

$-0.199$

(0.086)

(0.069)

$(0.085)$

(0.068)

(0.092)

(0.127)

Baseline Test Z-score

$0.076 * * *$

$0.063 * * *$

(0.020)

(0.016)

ABC*Post

2009 Cohort

0.041

0.081

(0.084)

(0.069)

Female

$-0.501 * * *$

$-0.506^{* * *}$

(0.044)

(0.044)

Age

$-0.013 * * *$

$-0.015 * * *$

0.025

(0.084)

$(0.016)$

Sub-region fixed effects

$$
\text { (0.002) }
$$

(0.002)

$-0.490 * * *$

(0.044)

$-0.013 * * *$

No

Yes

(0.002)

7,165

No

7,165

0.156

6,928

0.071

(0.069)

$-0.500 * * *$

(0.045)

$-0.015^{* * *}$

$0.696^{* *}$
$(0.316)$
$0.491 * * *$
$(0.146)$

$0.696^{* *}$

(0.309)

Number of observations

0.085

0.092

(0.002)

$-1.004 * * *$

(0.127)

$\mathrm{R}^{2}$

Notes: Each column represents a separate regression. Panel A presents results with writing test scores as the dependent variable. Panel B present results for math. Test-scores in Columns 1 through 4 are normalized based on the contemporaneous non-ABC distribution. Test scores in Columns 5 and 6 are normalized based upon the baseline non-ABC distribution. The sub-region is the level at which the $\mathrm{ABC}$ program was randomized. Huber-White standard errors cluster at the village level presented in parentheses. ${ }^{* * *}, * *,{ }^{*}$ denote statistical significance at the 1,5 and 10 percent levels, respectively. 
Table A4. Effects of the ABC Program by Year

\begin{tabular}{|c|c|c|}
\hline & (1) & $(2)$ \\
\hline \multicolumn{3}{|c|}{ Panel A: Writing Z-Scores } \\
\hline $\mathrm{ABC} *$ Post ( 1 year treatment $)$ & $\begin{array}{c}0.222 * * \\
(0.102)\end{array}$ & $\begin{array}{c}0.232 * * \\
(0.101)\end{array}$ \\
\hline $\mathrm{ABC} *$ Post (2 year treatment) & $\begin{array}{c}0.147 \\
(0.111)\end{array}$ & $\begin{array}{c}0.139 \\
(0.110)\end{array}$ \\
\hline Post (1 year treatment) & $\begin{array}{l}-0.001 \\
(0.070)\end{array}$ & $\begin{array}{c}-0.005 \\
(0.070)\end{array}$ \\
\hline Post (2 year treatment) & $\begin{array}{l}-0.009 \\
(0.074)\end{array}$ & $\begin{array}{c}-0.047 \\
(0.079)\end{array}$ \\
\hline $\mathrm{ABC}$ & $\begin{array}{l}-0.051 \\
(0.047)\end{array}$ & $\begin{array}{c}-0.051 \\
(0.048)\end{array}$ \\
\hline Gender, Age, Cohort & No & Yes \\
\hline Sub-region fixed effects & Yes & Yes \\
\hline Number of observations & 13,402 & 12,823 \\
\hline $\mathrm{R}^{2}$ & 0.033 & 0.086 \\
\hline \multicolumn{3}{|l|}{ Panel B: Math Z-Scores } \\
\hline ABC*Post (1 year treatment $)$ & $\begin{array}{c}0.228 * * \\
(0.105)\end{array}$ & $\begin{array}{c}0.244 * * \\
(0.108)\end{array}$ \\
\hline \multirow[t]{2}{*}{$\mathrm{ABC} *$ Post $(2$ year treatment $)$} & $0.297 * *$ & $0.293 * *$ \\
\hline & $\begin{array}{c}(0.134) \\
-0.002\end{array}$ & $\begin{array}{c}(0.133) \\
-0.008\end{array}$ \\
\hline Post (1 year treatment) & $(0.0790)$ & $(0.0805)$ \\
\hline \multirow[t]{2}{*}{ Post (2 year treatment) } & -0.010 & -0.078 \\
\hline & $(0.088)$ & $(0.093)$ \\
\hline \multirow{2}{*}{$\mathrm{ABC}$} & $-0.095^{*}$ & $-0.096^{*}$ \\
\hline & $(0.0548)$ & $(0.0545)$ \\
\hline Gender, Age, Cohort & No & Yes \\
\hline Sub-region fixed effects & Yes & Yes \\
\hline Number of observations & 13,420 & 12,840 \\
\hline $\mathrm{R}^{2}$ & 0.039 & 0.088 \\
\hline
\end{tabular}

Notes: Each column represents a separate regression. Panel A presents results with writing z-scores as the dependent variable. Panel B present results with math z-scores as the dependent variable. All test-scores are normalized to the contemporaneous non- $\mathrm{ABC}$ distribution. The sub-region is the level at which the $\mathrm{ABC}$ program was randomized. $* * *, * * *$ denote statistical significance at the 1,5 and 10 percent levels, respectively. Huber-White standard errors clustered at the village level are in parentheses. 
Table A5: Persistent Effects of the ABC Program: Bounding

\begin{tabular}{lcc}
\hline \hline Panel A: Writing Z-Scores & Lower Bound & Upper Bound \\
\hline & $(1)$ & $(2)$ \\
\cline { 2 - 3 } ABC*Post (January round) & 0.069 & $0.200^{* *}$ \\
& $(0.078)$ & $(0.077)$ \\
Gender, Age, Cohort & Yes & Yes \\
Sub-region fixed effects & Yes & Yes \\
Number of observations & 18615 & 18626 \\
$\mathrm{R}^{2}$ & 0.11 & 0.122 \\
\hline Panel B: Math Z-Scores & & \\
ABC*Post (January round) & $0.129 *$ & $0.272^{* * *}$ \\
& $(0.073)$ & $(0.073)$ \\
Gender, Age, Cohort & Yes & Yes \\
Sub-region fixed effects & Yes & Yes \\
Inverse Mills' Ratio & Yes & Yes \\
Number of observations & 18660 & 18660 \\
$\mathrm{R}^{2}$ & 0.104 & 0.108 \\
\hline
\end{tabular}

Notes: All test scores are normalized to the contemporaneous non-ABC distribution. Results include data collected 7 months after the end of classes for the 2009 and 2010 cohorts. The upper bound is constructed by dropping the highest test scores from the January round in the non-ABC villages. The lower bound is constructed by dropping lowest test scores from the January round in the $\mathrm{ABC}$ villages. All regressions include controls for the $\mathrm{ABC}$ program, the June test score round ("post"), the January test score round ("January post") and the interaction between the $\mathrm{ABC}$ program and the June test score round. The sub-region is the level at which the $\mathrm{ABC}$ program was randomized. Huber-White standard errors clustered at the village level in parentheses. ***, **, * denote statistically significance at 1, 5, 10 percent, respectively. 


\section{Table A6. Characteristics of Hotline Participants}

\begin{tabular}{lccc}
\hline \hline & & & \\
& Mean (s.d.) & Min & Max \\
\cline { 2 - 4 } Region $(0=$ Zinder, 1=Dosso) & $.187(.39)$ & 0 & 1 \\
Gender $(0=$ Male, $1=$ Female) & $.167(.37)$ & 0 & 1 \\
Cohort $(0=2010,1=2009)$ & $.575(.49)$ & 0 & 1 \\
Writing Test Score & $3.88(2.10)$ & 0 & 6 \\
Math Test Score & $3.40(1.37)$ & 0.5 & 6 \\
\hline Notes: Regressions include data from the call-in hotline between January and March 2011. & \\
\hline \hline
\end{tabular}

